\title{
Simulation as a tool for assessing and evolving your current personal protective equipment: lessons learned during the coronavirus disease (COVID-19) pandemic
}

\author{
Shannon L. Lockhart, MD, FRCPC · Justen J. Naidu, MD, \\ FRCPC · Charanjit S. Badh, MBBS, FRCPC, FRCA, FICM • Laura V. Duggan, MD, \\ FRCPC
}

Received: 18 March 2020/ Accepted: 18 March 2020/Published online: 27 March 2020

(C) Canadian Anesthesiologists' Society 2020

\section{To the Editor,}

We believe that protection of the well-being of healthcare providers while maintaining a workforce sufficient to respond to the coronavirus disease (COVID19) are fundamental to pandemic planning. In this letter, we describe how our hospital used low-fidelity airway simulation to assess and evolve the personal protective equipment (PPE) used for airway management of patients with COVID-19.

We have now had 47 healthcare workers processed through the COVID-19 airway simulation scenario. ${ }^{\mathrm{A}}$ During the first day of the simulation, we used our facility's recommended PPE comprising a "yellow gown" (Eden Textile micro fibre isolation gown, Edmonton, AB, Canada), a N95 respirator, a visor integrated into a surgical mask, and non-sterile nitrile gloves (Figure A). When the practitioners changed the simple face mask to allow bagmask ventilation during the induction of anesthesia, a patient cough was simulated. We did this by using Glo Germ $^{\mathrm{TM}}$ powder (Marlatek, Inc., Brockville, ON, Canada), a product designed to identify the simulated spread of microorganisms, which was brushed as powder onto the mannequin (contact) and forcefully expelled from the

\footnotetext{
S. L. Lockhart, MD, FRCPC ( $\varangle)$.

Department of Anesthesiology, St. Paul's Hospital, University of British Columbia, Vancouver, BC, Canada

e-mail: slockhart@providencehealth.bc.ca

J. J. Naidu, MD, FRCPCC. S. Badh, MBBS, FRCPC, FRCA, FICM

University of British Columbia, Vancouver, BC, Canada

\section{V. Duggan, MD, FRCPC}

Department of Anesthesiology \& Pain Medicine, University of Ottawa, Ottawa, ON, Canada
}

airway mannequin's naris using a MAD Nasal ${ }^{\mathrm{TM}}$ intranasal mucosal atomization device (Teleflex, Morrisville, NC, USA) (droplet). Spread of the simulated nasal secretions was visualized with ultraviolet light.

This technique revealed that the reusable yellow gowns were permeable to liquid; six out of the six first participants had visible soilage on their scrubs beneath their gowns. During the fifth simulation, an airway assistant wearing a disposable Association for the Advancement of Medical Instrumentation (AAMI) level-3 surgical gown (Sirus sterile nonreinforced surgical gown; Medline, Chicago, IL, USA) showed no contamination of scrubs beneath the gown. It also came to our attention that there was a significant amount of contamination on the practitioner's neck, on the base of their wrist, as well as on their lower pants and shoes.

While skin contamination is not a method of transmission for the severe acute respiratory syndrome coronavirus- 2 responsible for causing COVID-19, these areas of soilage increase the risk for self-contamination (e.g., during doffing) via mucous membranes. We approached our institution's Infection Control and Prevention Group with the findings of these first six participants; they indicated that the supplied yellow gowns lose their permeability protection after 25 washes.

We have since upgraded our PPE to disposable surgical gowns or coveralls (Kleenguard ${ }^{\mathrm{TM}}$ A60; Kimberly-Clark, Roswell, GA, USA) of at least an AAMI level-3 protection consistent with US Food and Drug Administration recommendations for moderate to highly invasive procedures. ${ }^{1}$ We have also included neck protection in the form of either a coverall, or if a surgical gown is worn,

\footnotetext{
A Available from URL: https://twitter.com/ShannonLockhart/status/ 1239032499994386433 (accessed March 2020).
} 
FIGURE Personal protective equipment (PPE) used during aerosol-generating medical procedures (AGMP). After assessing sites of contamination using simulation, we changed our originally recommended PPE (A) to include double highwrist surgical-type gloves, improved head and neck cover (surgical hood), and an Association for the Advancement of Medical Instrumentation (AAMI) level-3 gown (B)
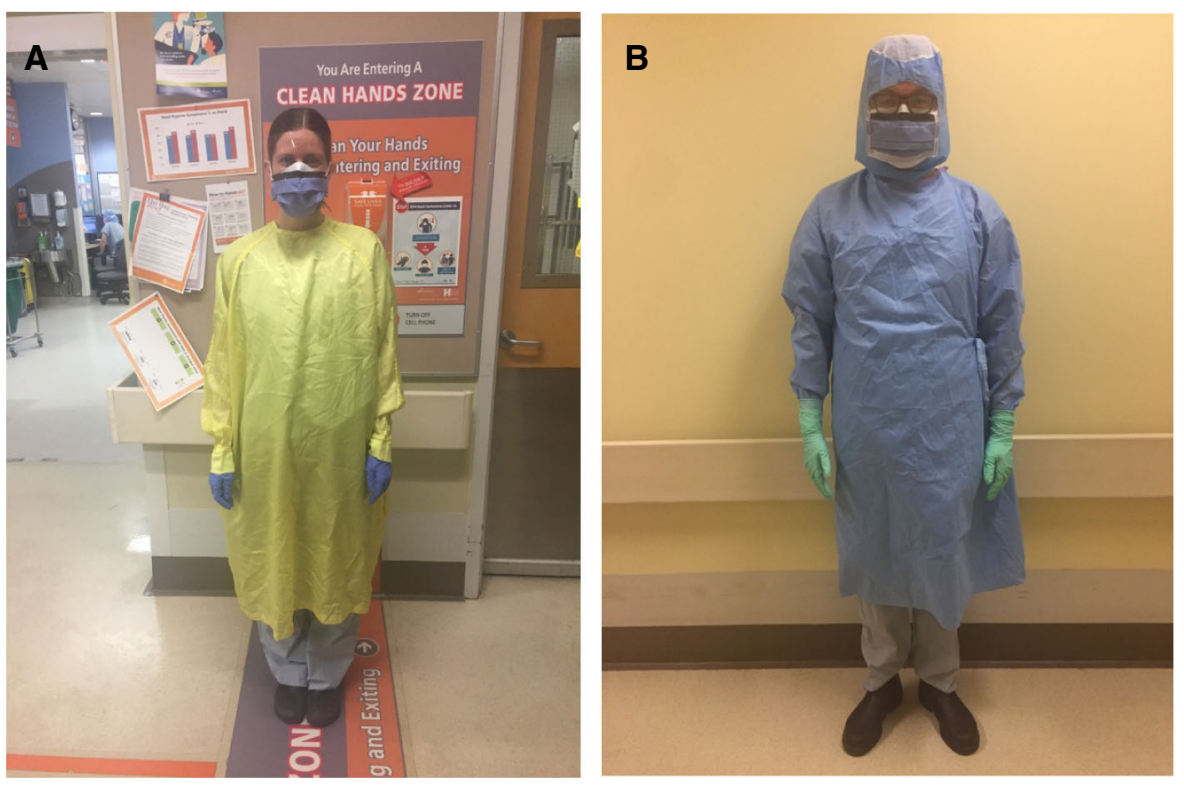

Originally recommended PPE for AGMP: Modified PPE for AGMP:

N95 respirator

Eye protection (surgical mask with visor)

Reusable yellow gown

(AAMI Level 2, prior to 25 washes)

Single pair of nitrile gloves

(no cuff specification)

No head covering; no shoe covering

\section{N95 respirator}

Eye protection (surgical mask with visor) Disposable surgical gown (AAMI Level 3) Double high-cuffed (surgical-type) gloves Surgical hood with ties (head and neck covering) Knee high shoe covering (not shown) a surgical hood with tie neck (priMed Medical Products Inc., Edmonton, AB, Canada) (Figure B). We now use high-cuff $(9-12 \mathrm{~cm})$ gloves, either non-sterile nitrile gloves or surgical gloves, and double glove. Neck contamination was almost eliminated after this change. Hand and forearm contamination was reduced by encouraging removal of rings and a full $20 \mathrm{sec}$ surgical scrub style of cleaning with an alcohol-based hand rub at each step of the doffing process. Shoe and lower leg contamination was reduced by using the coverall with booties. We also have a surgical gown with knee-high booties as an alternative. Above all, it made practitioners aware that, despite meticulous donning and doffing, contamination is still highly possible and that a shower after an aerosol-generating medical procedure is very prudent.

An additional finding has been how the doffing process must be specific to the combination of PPE chosen, and that it must be practiced. Doffing procedures, through the lens of avoiding personal contamination, were heavily reinforced by the presence of Glo Germ ${ }^{\mathrm{TM}}$ on the participant's face, neck, forearm, or shoes-i.e., each glowing spot could be a nidus for self-contamination and infection.

This simulation scenario also increased morale within our department and promoted buy-in to the practicing of meticulous donning and doffing. Simulation was a powerful tool to test and adapt PPE that has significantly enhanced provider safety compared with baseline recommendations. We hope that this tool will preserve one of the most valuable resources during a pandemic-our healthcare worker workforce.

\section{Conflicts of interest None.}

Funding statement None.

Editorial responsibility This submission was handled by Dr. Hilary P. Grocott, Editor-in-Chief, Canadian Journal of Anesthesia.

\section{Reference}

1. U.S. Food and Drug Administration. Medical Gowns. Available from URL: https://www.fda.gov/medical-devices/personalprotective-equipment-infection-control/medical-gowns (accessed March 2020).

Publisher's Note Springer Nature remains neutral with regard to jurisdictional claims in published maps and institutional affiliations. 